\title{
Hearing loss in systemic sclerosis - Clinically important, potentially treatable and often overlooked
}

\section{Editorial}

Systemic sclerosis (SSc) is one of the most complex systemic autoimmune diseases [1] characterized by cutaneous and visceral fibrosis, immune dysregulation and vasculopathy [2]. The interplay of these three processes determines the clinical and pathologic manifestations of the disease [1]. The core signs and symptoms of SSc are Raynaud's phenomenon, skin thickening and serum autoantibody production, but patients have different patterns of internal organ involvement with variable presentations and outcomes [3].

Depending on the extent of cutaneous fibrosis, SSc is classified into two major subtypes: limited cutaneous ( $\mathrm{lcSSc}_{\mathrm{c}}$ ) and diffuse cutaneous SSc $(\mathrm{dcSSc})$. In $\mathrm{dcSS}$, the presence of skin lesions is more extensive, internal organs involvement is relatively more severe [4] and it is typically associated with antitopoisomerase I (antiScl-70) antibodies, earlier age of onset and a poorer prognosis [5,6]. Pulmonary fibrosis and pulmonary arterial hypertension (PAH) are the two most feared complications, representing the major causes of mortality in SSc patients [7]. Owning to its complex nature and heterogeneity, SSc remains one of the greatest challenges in rheumatology [4]. Esophageal dysmotility is frequent in SSc patients and trigeminal nerve dysfunction may occur [8]. Even though cranial nerve involvement is an uncommon feature of this connective tissue disorder, and when it occurs it is the trigeminal nerve that is primarily affected, involvement of the eighth cranial nerve was reported in a lcSSc patient with sensorineural hearing loss (SNHL) [9]. The microangiopathy of SSc is felt to be primarily responsible for these neurological deficits and the deposition of fibrous tissue may be a secondary phenomenon and contribute to the process by compression of cranial nerves [10]. SSc is also associated with diminished interincisal distance that is related to the overall disease severity [11] and an increased frequency of xerophthalmia, cataract, increased intraocular pressure and fundus vascular abnormalities has been reported [12]. Ear involvement is not uncommon and should be taken into consideration during diagnostic and therapeutic procedures of SSc [13].

Autoimmune SNHL was originally defined by the presence of progressive hearing loss (with or without vertigo) and a positive response to corticosteroids [14]. Auto-immune SNHL can occur as one of the clinical features of systemic immune-mediated disorders as vasculitic syndromes, systemic lupus erythematosus, relapsing polychondritis and SSc or as a distinct clinical entity, the so-called auto-immune inner ear disease (AIED). The clinical evolution of the hearing loss during the course of the systemic disease is extremely variable, while the AIED is usually characterized by a rapidly developing (weeks or months) progressive hearing loss. A timely clinical assessment and treatment can positively affect the prognosis of the hypoacusia. No laboratory test will give a sure diagnosis of autoimmune hearing loss and only a good response to corticosteroids and/or immunomodulatory agents can indirectly confirm this diagnosis [15].

Ear involvement is frequent in SSc [13] and has been described in lcSSc [16] and dcSSc [17], where hearing loss and vestibulopathy coexist with other disease manifestations. The impairment of multiple vestibular endorgans points to peripheral vestibulopathy of ischemic origin or an intra-labyrinthine autoimmune process [16,18]. As in other manifestations of SSc, immunosuppressive therapy is effective in AIED when the disease is in the initial inflammatory phase as the fibrotic and ischemic phases are generally irreversible [19].

\author{
Tamer A. Gheita*1 \& Hanan M \\ Fathi $^{2}$ \\ 'Rheumatology Department, Faculty of \\ Medicine, Cairo University, Egypt \\ ${ }^{2}$ Rheumatology Department, Faculty of \\ Medicine, Fayoum University, Egypt \\ *Author for correspondence: \\ gheitamer@hotmail.com
}


The prevalence of auditory and vestibular dysfunction in SSc patients ranged from 20$77 \%$ with bilateral SNHL as the most frequent. Choclear vasculopathy has been postulated as a possible cause of hearing impairment in SSc [20]. Systemic sclerosis patients may develop hearing and balance disorders as a result of the immune-mediated vasculitis and fibrosis in the inner ear [13].

Hearing is usually examined subjectively by means of pure tone and speech audiometry. Additional objective examinations involve measuring of otoacoustic emissions generated in the inner ear cochlea or brain stem responses formed within the auditory pathway. Immittance measurements, used to evaluate the middle ear, include tympanometry probing the mobility of the tympanic membrane and middle ear pressure, and acoustic reflex assessing the functionality of the ossicular chain [21].

The clinical heterogeneity among patients remains poorly understood and critical gaps in understanding the biologic basis of SSc persist [22]. Auditory manifestations of connective tissue disorders represent a clinical diagnostic challenge as they often constitute the initial sign of an otherwise asymptomatic autoimmune disease. Moreover, in patients with known autoimmune rheumatic diseases, ENT manifestations can be overlooked [8].

The prevalence of bilateral SNHL among SSc patients ranges from $20 \%$ to $69 \%$, while a conductive component contributes to the hearing loss in $3-11 \%$ of them $[13,16,17]$. Hearing loss and vestibular derangement were reported in SSc patients with an established diagnosis and not as the presenting symptom of the disease. AIED with bilateral sudden SNHL has been reported as the initial symptom in lcSSc preceding the appearance of scleroderma skin changes [23]. As the diversity of systemic manifestations in SSc is directly related to the form of the disease subtype (limited or diffuse), it is hypothesized that auditory and vestibular changes also have these variations [20]. SSc disease appears to be directly responsible for audiovestibular damage as in $80 \%$ of patients with such involvement no other apparent cause could be revealed. SSc should be included among the autoimmune diseases which may cause audiovestibular disturbances [24].

A lower frequency of hearing loss was reported in $25 \%$ of SSc patients, mostly sensorineural affecting the middle and high pure tone frequencies [25] and further investigation was recommended for a better understanding of the mechanism of ear damage in scleroderma. In SSc patients, slightly higher auditory thresholds were reported 1 year after the onset of the disease with a significant aggravation after 2 years. Patients had bilateral absence of acoustic reflexes and of speech intelligibility [26]. Hearing damage in SSc patients first affects high-frequency hearing, and patients may not be aware of their hearing loss due to the severity of other manifestations of the disease. Inflammation of small vessels in the cochlear nerve and the reduced blood flow to the cochlea may be the beginning of the inner ear pathogenesis in autoimmune diseases [23]. The inner ear compromise, especially the cochlea, may be a consequence of SSc vasculopathy. The cochlea is a highly sensitive organ to blood changes, and the hypoxia resulting from vascular changes of the disease may lead to the death of hair cells [27]. Clinically, these changes are expressed by SNHL, which corroborates the findings that indicate lower prevalence of this type of hearing loss [20]. The sensorineural damage and hearing loss in SSc may be attributed to vasculitis, neuritis, or an ototoxic effect of the drugs used in its treatment [25] and at lower frequencies could be due to cochlear hydrops [28]. Interestingly, a normal tympanometry has been reported in SSc [26].

There is a possible role of vasculitis and neuritis in the development of SNHL in SSc patients [25]. Early recognition of neurological abnormalities in rheumatic diseases can help prevent permanent sensorimotor or cognitive function loss [29].

Hearing threshold may be associated with disease severity and further investigations are encouraged in spite no remarkable correlation was found between hearing loss with age, systemic manifestations of the disease, presence of autoantibodies, drug therapy, diseaseduration, type and severity of SSc disease [13,25].

SSc may cause subclinical SNHL before progressing to a clinical problem and thus it is recommended that these patients undergo audiometric tests. The likelihood that SNHL may be due to an underlying vasculopathy or neuritis mandates an increased awareness and clinical suspicion of hearing loss in SSc patients. Although rheumatologists can address hearing loss, the management of this aspect of SSc is often overlooked.

\section{Conflict of interest}

The authors report no declarations for conflict of interest. 


\section{References}

1. Pattanaik D, Brown M, Postlethwaite BC et al. Pathogenesis of systemic sclerosis. Front. Immunol. 6, 272 (2015).

2. Ramos PS, Silver RM, Feghali-Bostwick CA. Genetics of systemic sclerosis: recent advances. Curr. Opin. Rheumatol. 27(6), 521-9 (2015).

3. Gabrielli A, Avvedimento EV, Krieg TS. Scleroderma. N. Engl. J. Med. 360(19), 1989-2003 (2009).

4. Affandi AJ, Radstake TR, Marut W. Update on biomarkers in systemic sclerosis: tools for diagnosis and treatment. Semin. Immunopathol. 37(5), 475-87 (2015).

5. Steen VD. The many faces of scleroderma. Rheum. Dis. Clin. North. Am. 34, 1-15 (2008).

6. LeRoy EC, Black C, Fleischmajer R et al. Scleroderma (systemic sclerosis): classification, subsets and pathogenesis. J. Rheumatol. 15, 202-205 (1988).

7. Masi AT. Classification of systemic sclerosis (scleroderma): relationship of cutaneous subgroups in early disease to outcome and serologic reactivity. $J$. Rheumatol. 15, 894-8 (1988).

8. Papadimitraki ED, Kyrmizakis DE, Kritikos I et al. Earnose-throat manifestations of autoimmune rheumatic diseases. Clin. Exp. Rheumatol. 22(4), 485-94 (2004).

9. Iskandar SB, Loyd S, Roy TM. Cranial nerve VIII involvement in a patient with progressive systemic sclerosis. Tenn. Med. 97(3), 117-9 (2004).

10. Teasdall RD, Frayha RA, Shulman LE. Cranial nerve involvement in systemic sclerosis (scleroderma): a report of 10 cases. Medicine. 59(2), 149-59 (1980).

11. Baron M, Hudson M, Tatibouet S et al. Relationship between disease characteristics and orofacial manifestations in systemic sclerosis: Canadian Systemic Sclerosis Oral Health Study III. Arthritis. Care. Res. 67(5), 681-90 (2015).

12. Waszczykowska A, Gos R, Waszczykowska E et al. Prevalence of ocular manifestations in systemic sclerosis patients. Arch. Med. Sci. 9(6), 1107-13 (2013).

13. Maciaszczyk K, Waszczykowska E, Pajor A et al. Hearingorgandisorders in patients with systemic sclerosis. Rheumatol. Int. 31(11), 1423-8 (2011).

14. Loveman DM, de Comarmond C, Cepero R et al. Autoimmune sensorineural hearing loss: clinical course and treatment outcome Semin. Arthritis. Rheum. 34(2), 538-43 (2004).

15. Berrettini S, Ravecca F, Bruschini L et al. Progressive sensorineural hearing loss: immunologic etiology. Acta. Otorhinolaryngol. Ital. 18(4 Suppl 59), 33-41 (1998).
16. Amor-Dorado JC, Arias-Nunez MC, Miranda-Filloy $\mathrm{JA}$ et al. Audiovestibular manifestations in patients with limited systemic sclerosis and centromere protein-B (CENP-B) antibodies. Medicine. 87(3), 131-41 (2008).

17. Monteiro TA, Christmann RB, Bonfa E et al. Hearing loss in diffuse cutaneous systemic scleroderma. Scand. J. Rheumatol. 40(6), 467-71 (2011).

18. Amor-Dorado JC, Barreira-Fernandez MP, Arias-Nunez MC et al. Benign paroxysmal positional vertigo and clinical test of sensory interaction and balance in systemic sclerosis. Otol. Neurotol. 29(8), 1155-61(2008).

19. Buniel MC, Geelan-Hansen K, Weber PC et al. Immunosuppressive therapy for autoimmune inner ear disease. Immunotherapy. 1(3), 425-34 (2009).

20. Rabelo MB, Corona AP. Auditory and vestibular dysfunctions in systemic sclerosis: literature review. Codas. 26(5), 337-42 (2014).

21. Laitakari J, Kokkonen J. Functional examinations of the ear and auditory pathway. Duodecim. 127(8), 826-34 (2011).

22. Korman BD, Criswell LA. Recent advances in the genetics of systemic sclerosis: toward biological and clinical significance. Curr. Rheumatol. Rep. 17(3), 21 (2015).

23. Deroee AF, Huang TC, Morita N et al. Sudden hearing loss as the presenting symptom of systemic sclerosis. Otol. Neurotol. 30, 277-9 (2009).

24. Berrettini S, Ferri C, Pitaro N et al. Audiovestibular involvement in systemic sclerosis. ORL. J. Otorhinolaryngol. Relat. Spec. 56(4), 195-8 (1994).

25. Kastanioudakis I, Ziavra N, Politi EN et al. Hearing loss in progressive systemic sclerosis patients: a comparative study. Otolaryngol. Head. Neck. Surg. 124(5), 522-5 (2001).

26. Santarelli R, Monte PSED, Arslan EGE. Auditory neuropathy in systemic sclerosis: a speech perception and evoked potential study before and after cochlear implantation. Eur. Arch. Otorhinolaryngol. 263, 809-15 (2006).

27. Lazarini PR, Camargo AC. Idiopathic sudden sensorineural hearing loss: etiopathogenic aspects. Braz. J. Otorhinolaryngol. 72(4), 554-61 (2006).

28. Andonopoulos AP, Naxakis S, Goumas $\mathrm{P}$ et al. Sensorineural hearing disorders in systemic lupus erythematosus: a controlled study. Clin. Exp. Rheumatol. 13, 137-41 (1995).

29. Di Comite G. Sabbadini MG. Neurological involvement in rheumatological diseases. Neurological. Sciences. 26 Suppl 1, 9-14 (2005). 